\title{
Discs of planetary-mass objects in $\sigma$ Orionis
}

\author{
M. R. Zapatero Osorio ${ }^{1}$, J. A. Caballero ${ }^{2}$, V. J. S. Béjar ${ }^{1}$, R. Rebolo ${ }^{1,3}$, D. Barrado y Navascués ${ }^{4}$, G. Bihain ${ }^{1,3}$, \\ J. Eislöffel ${ }^{5}$, E. L. Martín ${ }^{1,6}$, C. A. L. Bailer-Jones ${ }^{2}$, R. Mundt ${ }^{2}$, T. Forveille ${ }^{7,8}$, and H. Bouy ${ }^{9,1}$ \\ 1 Instituto de Astrofísica de Canarias, 38200 La Laguna, Tenerife, Spain \\ e-mail: mosorio@iac.es \\ 2 Max-Planck-Institut für Astronomie, Königstuhl 17, 69117 Heidelberg, Germany \\ Consejo Superior de Investigaciones Científicas, Spain \\ LAEFF-INTA, PO Box 50727, 28080 Madrid, Spain \\ Thüringer Landessternwarte, Sternwarte 5, 07778 Tautenburg, Germany \\ ${ }^{6}$ University of Central Florida, Dept. of Physics, PO Box 162385, Orlando, FL 32816-2385, USA \\ 7 Canada-France-Hawaii Telescope Corporation, 65-1238 Mamalahoa Highway, Kamuela, HI 96743, Hawai'i, USA \\ Laboratoire d'Astrophysique, Observatoire de Grenoble, BP 53, 38041 Grenoble Cedex 9, France \\ 9 Astronomy Department, University of California, Berkeley, CA 94720, USA
}

Received 19 June 2007 / Accepted 13 July 2007

\section{ABSTRACT}

\begin{abstract}
Aims. We searched for infrared flux excesses of planetary-mass candidates in the $\sigma$ Orionis cluster ( $\sim 3 \mathrm{Myr}, \sim 350 \mathrm{pc}$ ).
Methods. Using $I J H K_{\mathrm{s}}$ data from the literature and the [3.6], [4.5], [5.8], and [8.0] IRAC images of the $\sigma$ Orionis cluster from the Spitzer Space Telescope public archives, we constructed colour-colour diagrams and spectral energy distributions from 0.8 to $8.0 \mu \mathrm{m}$ of cluster candidates fainter than $J=18.0 \mathrm{mag}$, i.e. the planetary-mass borderline for $\sigma$ Orionis.

Results. Infrared flux excesses are detected longward of $5 \mu \mathrm{m}$ in seven objects (S Ori 54, 55, 56, 58, 60, S Ori J053956.8-025315 and S Ori J053858.6-025228) with masses estimated in the range 7-14 $M_{\text {Jup }}$. Emission at shorter wavelengths (4.5 $\left.\mu \mathrm{m}\right)$ in excess of the photosphere is probably observed in S Ori 56 and S Ori J053858.6-025228. The faintest and least massive object, S Ori 60, exhibits flux excess only at $8 \mu \mathrm{m}$. We ascribe these infrared excesses to the presence of circumsubstellar warm discs, providing additional confirmation for the objects' membership of $\sigma$ Orionis. The observed incidence of inner discs around planetary-mass objects is $\geq 50 \%$, which is consistent with the measured inner disc frequency among cluster brown dwarfs and low-mass stars, suggesting that these objects share a common origin. However, there is a trend for the inner disc rate to increase with decreasing mass (from $10 M_{\odot}$ through the substellar domain), which may be due to a mass-dependent timescale for the dissipation of the interior discs.
\end{abstract}

Key words. Galaxy: open clusters and associations: individual: $\sigma$ Orionis - stars: planetary systems: protoplanetary disks stars: low mass, brown dwarfs

\section{Introduction}

Young isolated planetary-mass objects $\left(\leq 12-14 M_{\text {Jup }}\right)$ were photometrically discovered in the Orion Nebula (Lucas \& Roche 2000), $\sigma$ Orionis cluster (Zapatero Osorio et al. 2000) and IC 348 (Najita et al. 2000). Spectra of these objects were presented in Zapatero Osorio et al. (2000), Lucas et al. (2001), Martín et al. (2001) and Barrado y Navascués et al. (2001). Since then, the detection of young planetary-mass candidates with masses in the interval 3-14 $M_{\text {Jup }}\left(1 M_{\odot}=1047 M_{\text {Jup }}\right)$ has challenged current theoretical models of stellar and substellar formation because the theory of direct collapse and fragmentation of molecular clouds predicts a minimum mass cutoff between 1 and $15 M_{\text {Jup }}$ (e.g. Boss 2000; Whitworth \& Stamatellos 2006). It is not well established whether free-floating planetary-mass objects represent the natural extension of the initial mass function derived for stars and brown dwarfs.

Further insight into low-mass formation processes is provided by the detection of circum(sub)stellar discs. Evidence for accretion events in isolated planetary-mass candidates has been suggested from the observations of strong $\mathrm{H} \alpha$ emission lines (e.g. Zapatero Osorio et al. 2002a; Barrado y Navascués et al. 2001, 2002). Other groups have reported on the presence of discs (based on infrared flux excesses) around young, low-mass substellar objects (e.g., Luhman et al. 2005a,b; Allers et al. 2006), which suggests that free-floating planetary-mass objects may also be the hosts of discs. Recently, Mohanty et al. (2007) has claimed the likely existence of an edge-on disc around the planetary member of the system 2MASS J1207334-393254AB.

Here, we present the detection of infrared flux excesses in seven $\sigma$ Orionis planetary-mass candidates and derive their inner disc frequency. The $\sigma$ Orionis cluster is very young (3 Myr, Oliveira et al. 2002; Zapatero Osorio et al. 2002b), nearby $(\sim 352$ pc; Perryman et al. 1997), and is relatively free of extinction $\left(A_{V}<1 \mathrm{mag}\right.$; Lee 1968). Recently, using Spitzer data, Hernández et al. (2007) and Caballero et al. (2007) have derived the disc rate among cluster members from high-mass stars through the brown dwarf domain down to $15 M_{\text {Jup }}$. We will compare our results with their measurements.

\section{Photometric data}

The $\sigma$ Orionis cluster was observed in the four channels (3.6, 4.5, 5.8 and $8.0 \mu \mathrm{m}$ ) of the Infrared Array Camera (IRAC; Fazio et al. 2004) on board the Spitzer Space Telescope on 2004 October 9 under the Spitzer Guaranteed Time Observation 
Table 1. IRAC/Spitzer photometry (magnitudes) of $\sigma$ Orionis planetary-mass candidates with infrared excesses.

\begin{tabular}{|c|c|c|c|c|c|c|c|c|}
\hline Object & Sp.T. & $J$ & [3.6] & [4.5] & [5.8] & [8.0] & $I-J$ & $J-K_{\mathrm{s}}$ \\
\hline S Ori 54 & M9.5 & $18.00 \pm 0.05$ & $16.01 \pm 0.15$ & $15.97 \pm 0.16$ & $15.26 \pm 0.32$ & $14.45 \pm 0.52$ & $3.31 \pm 0.06$ & \\
\hline S Ori 55 & M9 & $18.22 \pm 0.10$ & $16.61 \pm 0.12$ & $16.27 \pm 0.13$ & $15.60 \pm 0.37$ & $14.87 \pm 0.38$ & $3.30 \pm 0.10$ & $1.22 \pm 0.14$ \\
\hline S Ori J053956.8-025315 & & $18.27 \pm 0.08$ & $16.55 \pm 0.13$ & $16.48 \pm 0.22$ & $15.88 \pm 0.46$ & $14.45 \pm 0.33$ & $2.98 \pm 0.08$ & $1.09 \pm 0.10$ \\
\hline S Ori 56 & L1/L0.5 & $18.44 \pm 0.10$ & $16.10 \pm 0.09$ & $15.89 \pm 0.10$ & $15.37 \pm 0.29$ & $14.86 \pm 0.39$ & $3.30 \pm 0.11$ & $1.35 \pm 0.14$ \\
\hline S Ori 58 & L0 & $18.60 \pm 0.10$ & $16.49 \pm 0.11$ & $16.40 \pm 0.14$ & $15.77 \pm 0.44$ & $14.86 \pm 0.55$ & $3.32 \pm 0.11$ & $1.73 \pm 0.14$ \\
\hline S Ori J053858.6-025228 & & $18.61 \pm 0.08$ & $16.29 \pm 0.11$ & $15.97 \pm 0.13$ & $15.83 \pm 0.50$ & $\geq 15.15$ & $3.58 \pm 0.09$ & $1.32 \pm 0.10$ \\
\hline S Ori 60 & L2/L5.5 & $19.05 \pm 0.10$ & $16.47 \pm 0.11$ & $16.59 \pm 0.18$ & $\geq 16.0$ & $14.85 \pm 0.42$ & $3.47 \pm 0.11$ & $1.75 \pm 0.14$ \\
\hline
\end{tabular}

programme \#37 by G. Fazio. Hernández et al. (2007) provide information on the area covered, data aquisition, exposure times and data reduction. We downloaded the processed images (in the form of a mosaic) using the Leopard software and performed a photometric analysis on them. The camera produces images with $F W H M \sim 1$ 1.7 from 3.6 to $8.0 \mu \mathrm{m}$. Aperture photometry was obtained for various single, bright sources all across the frames using daophot in $\mathrm{IRAF}^{1}$, with an aperture radius of $12^{\prime \prime}$ and a background annulus extending from 12 to $24^{\prime \prime}$. For the four IRAC bands we adopted zero-point magnitudes of $17.30(3.6 \mu \mathrm{m}), 16.82(4.5 \mu \mathrm{m}), 16.33(5.8 \mu \mathrm{m})$ and $15.69 \mathrm{mag}$ $(8.0 \mu \mathrm{m})$. We derived the point spread function (PSF) from the brighter sources and applied this to determine photometry for $\sigma$ Orionis planetary-mass candidates known in the literature (Zapatero Osorio et al. 2000; González-García et al. 2006; Caballero et al. 2007). All of them have $J \geq 18$ mag, which corresponds to a maximum mass of $14 M_{\text {Jup }}$ at the age and distance of the cluster according to the models by Baraffe et al. (2002).

Six free-floating planetary-mass candidates are detected at $8.0 \mu \mathrm{m}$ with a significance between 4.5 and $6 \sigma$ when the peak of the detection is compared to the background. This implies that our final photometry is affected by relatively large error bars, but detections are reliable and daophot could manage to fit a PSF to determine observed magnitudes. The six objects are indicated in Table 1. Many other candidates remain undetected at $8.0 \mu \mathrm{m}$. Of the six, all except for S Ori 60 (the faintest object) are seen at $5.8 \mu \mathrm{m}$ with similar signal-to-noise ratio. Two more objects, S Ori J053858.6-025228 and S Ori J054007.0-023604, show flux emission at $5.8 \mu \mathrm{m}$. The [3.6] and [4.5] IRAC images are deeper than those taken at longer wavelengths and all planetary-mass candidates within the field of view of Spitzer are clearly detected. Our IRAC photometry is provided in Table 1, where only objects with excesses are indicated.

We compiled $I J H K_{\mathrm{s}}$ magnitudes from the literature (Zapatero Osorio et al. 2000; Martín et al. 2001; Caballero et al. 2007). The $I$ and $J$ magnitudes are available for all seven sources in Table 1. Planetary-mass candidates were typically selected from optical $(I)$ and near-infrared $(J)$ colour-magnitude diagrams; no particular bias toward disc-bearing objects is thus expected. Unfortunately, a few objects lack $H$ and/or $K_{\text {s }}$ photometry. The $I-J$ and $J-K_{\mathrm{s}}$ colours are listed in Table 1. Figure 1 illustrates the location of our seven objects in the $J$ vs. $J-[4.5]$ colour-magnitude diagram. To complete the $\sigma$ Orionis cluster sequence, we plotted the photometry of low-mass stars, brown dwarfs and planetary-mass objects from Caballero et al. (2007) and some unpublished data from our archives. Superimposed on the data is the 3-Myr isochrone from Baraffe et al. (2002), where theoretical $T_{\text {eff }}$ and luminosities were converted into

1 IRAF is distributed by National Optical Astronomy Observatories, which is operated by the Association of Universities for Research in Astronomy, Inc., under contract to the National Science Foundation, USA.

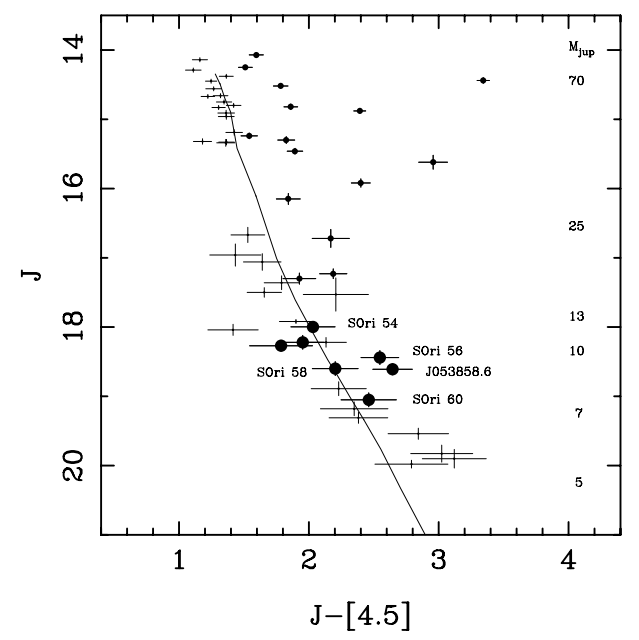

Fig. 1. Colour-magnitude diagram of low-mass $\sigma$ Orionis member candidates. Our objects with flux excesses at 5.8 and $8.0 \mu \mathrm{m}$ are plotted as large filled circles (some are labelled). Cluster members with flux excesses reported in Caballero et al. (2007) are indicated with filled circles of intermediate size. Masses in Jovian units are given on the right hand side. Overplotted with a solid line is the 3-Myr isochrone from Baraffe et al. (2002, see text). Current IRAC/Spitzer data cannot provide strong constraints on the presence of flux excesses below $J=18.5$ mag.

observables using colour-temperature and colour-bolometric corrections from Dahn et al. (2002), Vrba et al. (2004) and Patten et al. (2006). According to these models, our planetary-mass candidates have probable masses in the range 7 to $14 M_{\mathrm{Jup}}$.

Low-resolution spectra are available for five of the seven objects (Zapatero Osorio et al. 2000, 2002a; Martín et al. 2001; Barrado y Navascués et al. 2001); we provide their spectral types in Table 1. All candidates are classified as late-M to mid-L sources. S Ori 60 was typed at both optical and nearinfrared wavelengths. Its near-infrared spectral type is 3.5 subtypes cooler than the optical typing; this could be due to data noise and/or to the effects of a low-gravity atmosphere. Interestingly, S Ori 55 shows strong and variable $\mathrm{H} \alpha$ emission with pseudoequivalent widths ranging from $\sim 5$ to $410 \AA$ (Zapatero Osorio et al. 2002a). S Ori 54, 56, 58, and 60 have moderate $\mathrm{H} \alpha$ emission (if any) with pseudoequivalent widths below $30 \AA$ (Barrado y Navascués et al. 2001).

\section{Flux excesses and spectral energy distributions}

To search for mid-infrared flux excesses in the IRAC/Spitzer data, we constructed colour-colour diagrams for $\sigma$ Orionis member candidates with $I-J$ and $J-K_{\mathrm{s}}$ colours typical of mid-M types and cooler. The [3.6]-[5.8] and [3.6]-[8.0] indices are good indicators of flux excesses at wavelengths longer than $5 \mu \mathrm{m}$. Figure 2 depicts the latter index as a function of 


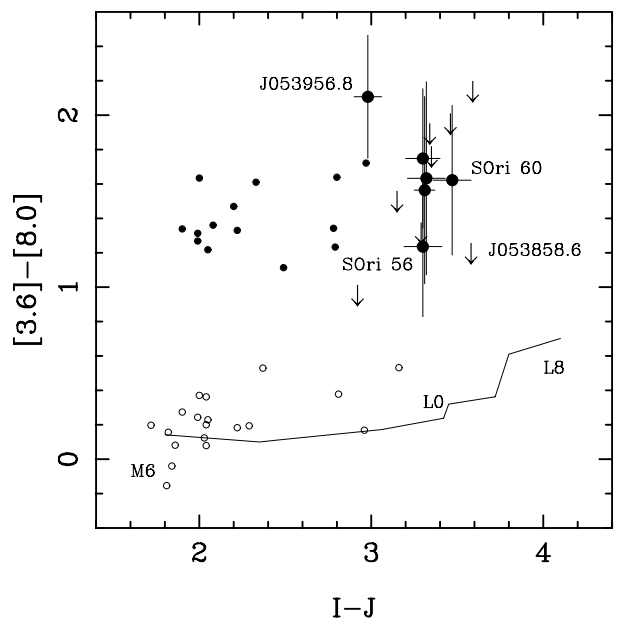

Fig. 2. Colour-colour diagram of $\sigma$ Orionis low-mass member candidates. Objects with flux excesses at 5.8 and $8.0 \mu \mathrm{m}$ are indicated with filled circles; objects with no IRAC/Spitzer flux excesses are depicted with open circles. The planetary-mass objects of Table 1 are shown with large filled circles (some are labeled). Arrows (upper limits) stand for cluster planetary-mass candidates that remain undetected in the [8.0]-band. Overplotted (solid line) is the field sequence of discless late-M and L dwarfs. Except for our objects, error bars are avoided for clarity.

the $I-J$ colour. Hartmann et al. (2005), Guieu et al. (2007), and Luhman et al. (2007) showed that disc-bearing objects in their Taurus samples exhibit colours of [3.6]-[5.8] $>0.4 \mathrm{mag}$ and [3.6]-[8.0] $>0.8 \mathrm{mag}$. All the disc-bearing $\sigma$ Orionis members of Caballero et al. (2007) depicted in Fig. 2 have redder [3.6]-[8.0] indices and lie far from the location of discless cluster members.

Therefore, to identify $\sigma$ Orionis planetary-mass objects with flux excesses, we applied these criteria to all candidates with $J \geq$ 18.0 mag. Despite their relatively large error bars, all seven objects in Table 1 exhibit flux excesses at 5.8 and/or $8.0 \mu \mathrm{m}$. Two out of the seven, namely S Ori 56 and S Ori J053858.6-025228, seem to show flux excesses at $4.5 \mu \mathrm{m}$ as well (see Fig. 3). Their red mid-infrared colours are indicative of disc-bearing objects. This interpretation provides further evidence for the objects being young and hence members of the 3-Myr $\sigma$ Orionis cluster.

The strong $\mathrm{H} \alpha$ emission observed in S Ori 55 is very probably related to gas accretion from a surrounding disc. The remaining objects with optical spectra do not appear to emit intensively in $\mathrm{H} \alpha$, suggesting that their accretion phase is fading away at the age of the cluster. Based on these $\mathrm{H} \alpha$ results, the frequency of accretion events is about $20 \%$ in the planetary-mass domain of $\sigma$ Orionis. We note that the IRAC/Spitzer colours of S Ori 55 do not differ significantly to within $1 \sigma$ of the photometric uncertainty from those of the non-accretors.

Spectral energy distributions from 0.85 up to $8.0 \mu \mathrm{m}$ were built for all seven free-floating planetary-mass objects. Results are shown in Fig. 3, where $\lambda F_{\lambda}$ is depicted as a function of wavelength. To convert magnitudes into fluxes we used the zero magnitude fluxes of Allen (2000) for the optical and near-infrared, and the zero magnitude fluxes given in the IRAC Data Handbook for the Spitzer data $(280.9,179.7,115.0$, and 64.1 Jy for [3.6], [4.5], [5.8], and [8.0], respectively). For comparison, overplotted in Fig. 3 are the typical energy distributions of late-M, early-L and mid-L field dwarfs obtained by averaging the normalized flux data by Patten et al. (2006), Liebert \& Gizis (2006) and
2MASS near-infrared magnitudes. These represent the photospheric emission expected for a given $T_{\text {eff }}$.

With few exceptions, our data points from 0.85 up to $4.5 \mu \mathrm{m}$ are well matched by discless field dwarfs with spectral types coincident within 2 subtypes with those from the literature. Regarding the two objects with no previous spectral type assignments, we derive M8 for S Ori J053956.8-025315 and L2 for S Ori J053858.6-025228 (uncertainty of \pm 2 subtypes). We note that the spectral energy distribution of the latter object is also compatible to within the observed error bars with spectral type L4, in which case we do not claim any flux excess detection at $5.8 \mu \mathrm{m}$ for it.

In our study, $\sigma$ Orionis planetary-mass object inner disc excess emission becomes clearly detectable longward of $5 \mu \mathrm{m}$. This appears to contrast with observations of more massive discbearing brown dwarfs and low-mass stars of $\sigma$ Orionis and other star-forming regions, where excess emission can be observed at shorter wavelengths (e.g. Guieu et al. 2007). Larger samples and better photometric precision are required for a proper comparison. We note that the faintest and least massive object with a disc in our data, S Ori $60\left(\sim 7-8 M_{\text {Jup }}\right)$, exhibits an excess only at $8 \mu \mathrm{m}$, while its emission from 0.85 up to $5.8 \mu \mathrm{m}$ is mainly photospheric.

\section{Disc frequency}

To determine the fraction of $\sigma$ Orionis isolated planetarymass objects that posses inner discs, we have to compare the seven "detections" with the total number of cluster candidates in the same mass (or magnitude) interval (14-7 $M_{\text {Jup }}, J \sim$ 18-19.3 mag). As shown in Fig. 1, there are twelve such candidates. Thus, we find that seven out of twelve (or six out of twelve if we discard S Ori J053858.6-025228, i.e. $50 \pm 20 \%$ ) $\sigma$ Orionis planetary-mass objects harbour discs. However, this number may represent a minimum fraction. On the one hand, we expect a few contaminants in our list of planetary-mass candidates (Caballero et al. 2007), which would be discless cluster non-members and should be excluded from the statistics. On the other hand, the IRAC/Spitzer data do not provide strong constraints on the presence of discs around the five candidates that remain undetected in the [8.0]-band (see the [3.6]-[8.0] upper limits in Fig. 2). We cannot therefore discard a disc frequency much higher than $50 \%$ among $\sigma$ Orionis free-floating planetarymass members.

The ratio $\geq 50 \%$ compares reasonably well with that obtained for cluster brown dwarfs with masses in the range 75-15 $M_{\text {Jup }}$ (47 $\pm 15 \%$, Caballero et al. 2007; $33.3 \pm 9.7 \%$, Hernández et al. 2007). However, there is a hint for the rate of disc-bearing objects increasing toward lower mass. Figure 4 shows the disc fraction determination for various mass ranges, from massive stars to the planetary regime of $\sigma$ Orionis. For a proper comparison with our statistics, we have drawn data from the literature based on IRAC/Spitzer photometric excesses. Massive stars present a lower disc frequency than low-mass stars and substellar objects. A similar result was found for IC 348 and the Upper Scorpious OB Association by Lada et al. (2006), Carpenter et al. (2006) and Scholz et al. (2007). This may suggest that, under the assumption of coevality, the timescales for inner disc evolution is longer for the least massive bodies. Deeper Spitzer data and a larger number of observations of young free-floating planets are required to confirm this.

Acknowledgements. This work is based in part on observations made with the Spitzer Space Telescope, which is operated by the JPL, Caltech under a contract 

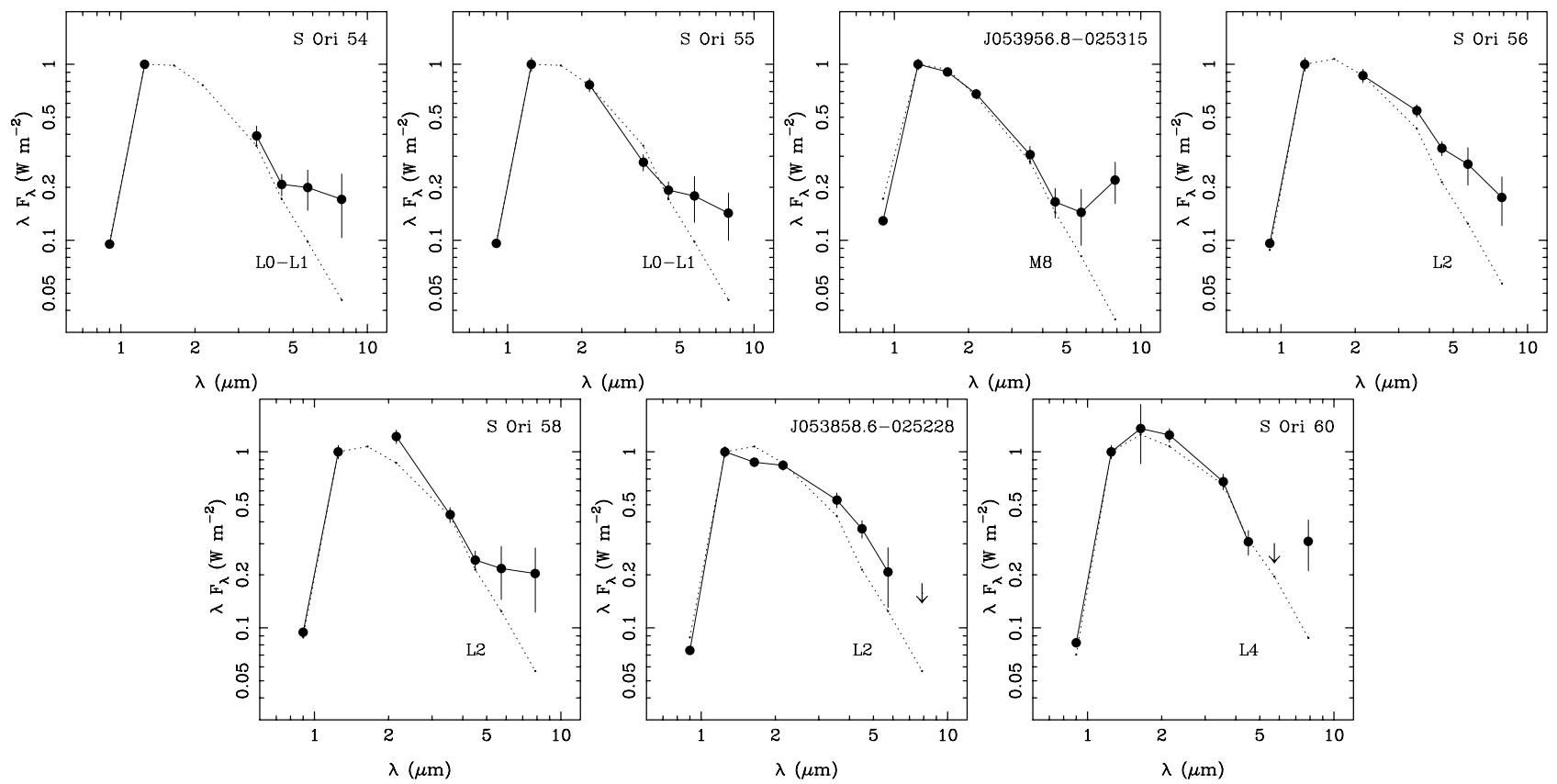

Fig. 3. Spectral energy distribution $\left(I J H K_{\mathrm{s}}\right.$, [3.6], [4.5], [5.8], and [8.0], solid line, filled circles) of the seven $\sigma$ Orionis isolated planetary-mass objects for which IRAC/Spitzer flux excesses have been detected. Upper limits are indicated with arrows. All data are normalized to 1.0 in the $J$-band. For comparison purposes, the average energy distributions of discless M8 to L4 field dwarfs are overplotted with dotted lines.

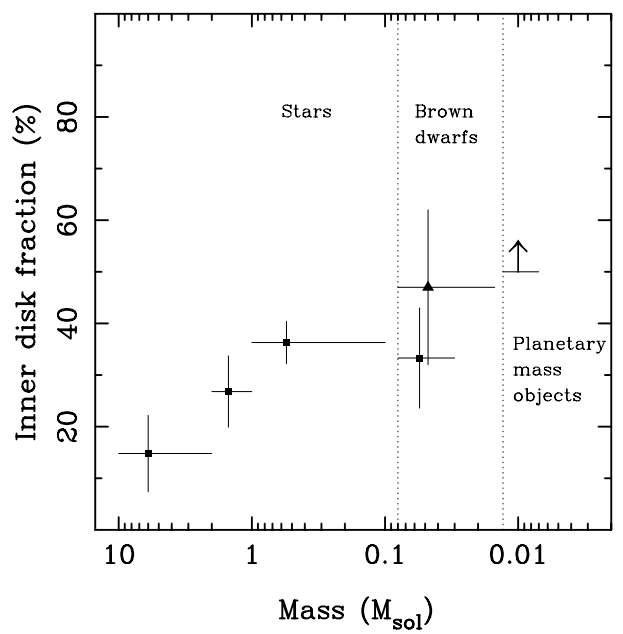

Fig. 4. Inner disc frequency of $\sigma$ Orionis stars, brown dwarfs and planetary-mass objects as a function of mass. Measurements from Hernández et al. (2007) and Caballero et al. (2007) are plotted as squares and triangles, respectively. Our determination for the planetarymass regime of the cluster is indicated with an arrow (lower limit, see text). Note that all measurements are obtained from the analysis of IRAC/Spitzer data. Horizontal error bars account for mass intervals.

with NASA. This research has made use of the SIMBAD database, operated at CDS, Strasbourg, France, and has been supported by the Spanish project AYA2006-12612. We thank T. Mahoney for the language revision. J.A.C. is an Alexander von Humboldt Fellow at the MPIA.

\section{References}

Allen, C. W. 2000, Astrophysical Quantities, 4th Ed. (New York: SpringerVerlag)

Allers, K. N., Kessler-Silacci, J. E., Cieza, L. A., \& Jaffe, D. T. 2006, ApJ, 644, 364
Baraffe, I., Chabrier, G., Allard, F., \& Hauschildt, P. H. 2002, A\&A, 382, 563 Barrado y Navascués, D., Zapatero Osorio, M. R., Béjar, V. J. S., et al. 2001, A\&A, 377, L9

Barrado y Navascués, D., Zapatero Osorio, M. R., Martín, E. L., et al. 2002, A\&A, 393, L85

Boss, A. P. 2000, ApJ, 536, L101

Caballero, J. A., Béjar, V. J. S., Rebolo, R., et al. 2007, A\&A, in press

Carpenter, J. M., Mamajek, E. E., Hillenbrand, L. A., \& Meyer, M. R. 2006, ApJ, 651, L49

Dahn, C. C., Harris, H. C., Vrba, F. J., et al. 2002, AJ, 124, 1170

Fazio, G. G., Hora, J. L., Allen, L. E., et al. 2004, ApJS, 154, 10

González-García, B. M., Zapatero Osorio, M. R., Béjar, V. J. S., et al. 2006, A\&A, 460, 799

Guieu, S., Pinte, C., Monin, J.-L., et al. 2007, A\&A, 465, 855

Hartmann, L., Megeath, S. T., Allen, L., et al. 2005, ApJ, 629, 881

Hernández, J., Hartmann, L., Megeath, T., et al. 2007, ApJ, 662, 1067

Lada, C. J., Muench, A. A., Luhman, K. L., et al. 2006, AJ, 131, 1574

Lee, T. A. 1968, ApJ, 152, 913

Liebert, J., \& Gizis, J. E. 2006, PASP, 118, 659

Lucas, P. W., \& Roche, P. F. 2000, MNRAS, 314, 858

Lucas, P. W., Roche, P. F., Allard, F., \& Hauschildt, P. H. 2001, MNRAS, 326, 695

Luhman, K. L., D'Alessio, P., Calvet, N., et al. 2005a, ApJ, 620, L51

Luhman, K. L., Adame, L., D’Alessio, P., et al. 2005b, ApJ, 635, L93

Luhman, K. L., Whitney, B. A., Meade, M. R., et al. 2007, ApJ, 647, 1180

Martín, E. L., Zapatero Osorio, M. R., Barrado y Navascués, D., Béjar, V. J. S., \& Rebolo, R. 2001, ApJ, 558, L117

Mohanty, S., Jayawardhana, R., Huélamo, N., \& Mamajek, E. 2007, ApJ, 657, 1064

Najita, J. R., Tiede, G. P., \& Carr, J. S. 2000, ApJ, 541, 977

Oliveira, J. M., Jeffries, R. D., Kenyon, M. J., Thompson, S. A., \& Naylor, T. 2002, A\&A, 382, L22

Patten, B. M., Stauffer, J. R., Burrows, A., et al. 2006, ApJ, 651, 502

Perryman, M. A. C., Lindegren, L., Kovalevsky, J., et al. 1997, A\&A, 323, L49

Scholz, A., Jayawardhana, R., Wood, K., et al. 2007, ApJ, 660, 1517

Vrba, F. J., Henden, A. A., Luginbuhl, C. B., et al. 2004, AJ, 127, 2948

Whitworth, A. P., \& Stamatellos, D. 2006, A\&A, 458, 817

Zapatero Osorio, M. R., Béjar, V. J. S., Martín, E. L., et al. 2000, Science, 290, 103

Zapatero Osorio, M. R., Béjar, V. J. S., Martín, E. L., Barrado y Navascués, D., \& Rebolo, R. 2002a, ApJ, 569, L99

Zapatero Osorio, M. R., Béjar, V. J. S., Pavlenko, Ya., et al. 2002b, A\&A, 384, 937 